\begin{tabular}{|c|}
\hline Between The Species \\
Issue IX \\
August 2009 \\
http://lla.calpoly.edu/bts/
\end{tabular}

\title{
The Man Who Mistook His Meal for a Hot Dog
}

H. Peter Steeves

DePaul University

When I was seven years old my father left us. For my mother it was the end of the world. For me and my brother, less so. He left on March 15 of that year, and by September-the month of my birthday-it was clear he was not coming back. My grandfather-who smoked Tiparillos, hated most of the people around him, and who I saw smile only three times in my life-took the opportunity to take me, my brother, and my mother along with my grandmother on their yearly vacation two hours south to Cincinnati. Cincinnati was The Big City. At the time it was also the home of The Big Red Machine, and so we saw a Cincinnati Reds baseball game (in which they lost to St. Louis) and went to eat at a nice restaurant that Saturday night. I smiled, perhaps, for the first time in six months.

Pop ordered for me that night and made a big deal of it. Before I could ask for my standard cheeseburger, he told the waiter that I would have a filet mignon, medium rare, with a baked potato. I said nothing. At the time it seemed as if hours passed. I had no idea what my plate would hold, what to expect when the waiter finally returned, and so I filled the minutes in fear, assuming it would be something terrible-probably with tentacles or maybe tasting vaguely of dirt-and I would have to eat it and pretend that everything was O.K. so as not to ruin this perfect trip.

When the steak finally arrived, I had never seen such a thing of beauty. The piece of meat looked like it had been sculpted from butter, and it floated-wrapped in bacon-on a life-raft of thick toast, bobbing in a sea of juices that smelled like...heroism. Pop leaned over to me and whispered: "You're the man of the house now. It all rests on your shoulders."

In college, I became a vegetarian. And a couple of years ago I had my first fake filet mignon. It was homemade, though based on a soy-based product called Stakelets sold by Worthington. It was incredibly delicious, wrapped up in soy bacon with a cup of beef-flavored juice on the side. And all I could think about was Pop-long-gone, dying, just as he had predicted, right before his retirement-who 
would surely think I had become a fake man.

\section{Vegetarian Filet Mignon}

1 box ( 4 patties) of Stakelets, thawed 1/ 2 cup of grated parmesan cheese 1/ 4 cup of Italian seasoned breadcrumbs 1 teaspoon of garlic powder 1 teaspoon of onion powder 1 teaspoon of dried oregano 1 teaspoon of dried parsley 1/4 teaspoon of black pepper (or to taste) 11/2 tablespoons of A-1 sauce 1 teaspoon of vegetarian Worcestershire sauce 2 eggs, slightly beaten 4 vegetarian bacon strips 1 onion (sliced vertically) 1 cup of sliced mushrooms 1/ 4 cup of red wine 1/ 4 cup of vegetarian beef flavored broth 2 slices of bread, cut thickly

In a food processor, coarsely grind the Stakelets. In a large bowl, thoroughly mix the Stakelets, parmesan cheese, breadcrumbs, garlic powder, onion powder, oregano, parsley, and black pepper. Add the A-1 sauce, Worcestershire sauce, and the eggs. Mix until all of the ingredients are moist. Divide the mixture and make two round, thick patties. Cook for about 3 minutes in the microwave until they achieve a semi-solid consistency. (Do not cook completely!) Lightly brown the bacon strips in a frying pan with oil, making sure that the strips are still flexible. Wrap each filet mignon patty with 2 bacon strips and secure them with 2 toothpicks. On low to medium heat, brown the patties in the same frying pan until fully cooked (about 20 minutes). In a separate pan, fry the onions and mushrooms, then add the red wine and beef broth and let the mixture cook until it starts to boil. Salt and pepper to taste. Toast the bread in the oven. To serve, place the filet mignons on the toast and cover with the onion and mushroom sauce.

Recipe by Marinés Fornerino

Animal representation routinely takes place in politics, ethics, and art. Someone, or something, stands in for the nonhuman animal, and the animal is re-presented to our consciousness-presented anew but altered, much like a memory. Politically and ethically, without truly knowing the Other such representation is impossible. We cannot make re-present what has never been present to us in the first place. This is, perhaps, the major theoretical failure of American representative democracy-and often the failure of animal rights arguments as well. We cannot represent and thus make re-present what we do not know, what has never been present for us. But our democratic ideals and much of our ethics are based on this.

Phenomenologically speaking, when I order for us at dinner, for instance, I am attempting to make present you, your will, and your perspective on our common Good. If I say "We will have coffee with dessert" then I am making a claim in first-person-plural that represents you by attempting to represent your will, to make it present in its absence. Edmund Husserl's term for this is apperception: a making co-present, there too but not there, that which is present in its absence. This can misfire. It misfires when I do not reflect your will accurately. It necessarily misfires if I do not first know your will. It also misfires if I do not have the right to speak for you and make your will present. The first 
question we should ask when considering giving voice to other beings, then, is whether or not this giving voice is always good or even possible. We do not want the giving of voices to become a ventriloquism act; we do not want to fail in our attempt to apperceive the common Good and speak for others on their behalf when our re-presentations of them are, from the get-go, so often out of whack.

All of this is important, I think, but I want to come at this point tangentially-to come at it by way of investigating the manner in which animals-and especially animal flesh-is made present in the medium of soy and other fake meat products for vegetarians. It is, perhaps, no new claim to say that meat represents various things in our culture-that it is tied to misogyny, violence, colonialism, racism, and all sorts of nasty things. It is no accident that my grandfather associated my first steak with my manhood-and a manhood, at that, which seemed based on denying my own mother full subjectivity. But let us begin with a simpler question, one that might even be somewhat more novel: is it in principle immoral to eat fake meat?

Most people would likely say that the obvious answer to such a question is no, assuming that the reason for one's vegetarianism is either the utilitarian worry about the pain and suffering of animals or the deontological worry about respecting an animal life as an end-in-itself. If no animal is actually harmed to make a vegetarian meat substitute, then no animal's life is actually disrupted or disrespected. One is merely eating soybeans or mushrooms or whatever sort of vegetable or grain from which the vegetarian food is created. These things may be dressed up to look like meat, but they are just vegetables and grains.

One initial response, however, might be that there is, indeed, something special about the fact that the food is dressed up to look like meat, and that the potential immorality of the act lies not in the eating of a veggie burger, for instance, but in the desire to eat the regular burger which the veggie burger is made to represent. It is a desire that is still present in the veggie burger. The craving is for animal flesh. As an enactment of that desire, then, eating the veggie burger would be troublesome. In fact, the more accurate the representation-the more the vegetarian substitutelooks, tastes, and smells like meat-the greater the success of the product. Is there not something bad about the desire itself, apart from whether or not any animal is affected?

Consider, for example, a parallel substitution. Pedophilia typically both harms and disrespects the children involved. But imagine we were to develop a pedophilia videogame in order to sublimate those desires-an explicit videogame in which the goal is to seduce, abduct, and rape children. 
In the world of anime, it sometimes seems as if we are already at this point. And indeed, last November, British authorities discovered a corner of a popular virtual world that seems to have been created specifically for the rape of virtual children. "Second Life" is an Internet site with over nine million users in Great Britain. Those who sign up create an avatar, interact with other users, and help to build a world together. One part of the world that they have built lies behind a secret wall in a shopping mall. Here, there is a playground filled with elementary school children who are ready to have sex with the users. Lawyers for the individuals who created and frequent the area within this virtual world have maintained that no laws have been broken. The British government agrees, but is still trying to find a way to prosecute.

Let's imagine that our videogame, then, is extremely graphic. In fact, the more lifelike it is, the better. Let's imagine that programmers are getting better and better at simulating the real thing, and as a result it is getting more and more difficult to tell the difference between an actual molestation and a virtual one. The thrills, the sensations, the physical and emotional rewards are all there for the pedophile. Yet no children are being harmed by the game, no lives are being disrespected and no sadness is being generated. On the contrary, there are a lot of people who might actually be very happy about the existence of such a game. It would allow the silencing of the harmful desire, or at least the redirection of it in such a way that it would no longer harm real children. There might even be less child molestation in the world. And yet, something seems questionable about the morality of all this.

How is fake meat any different? The closer it gets to being like the real thing, the more successful it is. There might even be fewer animals being harmed because the fake meat is so tasty. Is this, then, the only thing that matters?

I am a fan of fake meat. I eat it everyday if I can. And I have only started asking these questions of myself. I enjoy a fake turkey for Thanksgiving, for instance, and I wish that I did not have the desire at all. My palate is even too demanding to accept Tofurkey. I need to have something that approximates the turkey experience even better. I like it with stuffing and gravy and cranberry sauce. But how far do we want to take the simulation?

Imagine that Morningstar Farms were to come out with a new "turkey dinner experience." The turkey, sculpted entirely from soy but tasting completely like the real thing, would come complete with internal tubes filled with red-tinted corn syrup to simulate blood. Synthetic feathers would cover the body of the tofu-bird, and Disney Imagineers would add audio-animatronics so that you could 
power up the fake turkey and have it run around your back yard. Part of the preparation of the meal, then, would involve catching the fake bird, chopping its head off, draining the fake blood, defeathering the carcass, removing the gummi-organs inside, stuffing it, and roasting it up. J ust how far does the simulacra go before we judge it to have moral content of its own?

In the movie "The Matrix" (1999, Dir. Larry and Andy Wachowski), J oe Pantoliano's character, Cypher, agrees to sell out his friends and be re-inserted into the fake world of simulation in part because he is sick of living in the real world where life is hard and food tastes disgusting. He agrees to the traitorous act while eating a virtual steak, proclaiming that he doesn't care that it is not real, he loves how it seems to taste! The fake-meat eater is the villain of the story. Am I the villain as well?

I spend time and energy trying to get fake meat to taste more like the real thing. Taking a storebought vegetarian product and finding a way to make it even more like the real thing is, I think, one of the greatest skills imaginable.

\section{Vegetarian Cornish Hens}

1 10.6oz box (4 patties) of Quorn chik'n patties 1 tablespoon of vegetarian chicken flavored powdered broth 1 teaspoon of poultry seasoning $1 / 2$ teaspoon of garlic powder $1 / 2$ cup of grated parmesan cheese 1/ 4 cup of Italian seasoned breadcrumbs 2 eggs, slightly beaten 1 box of Stovetop (pork flavor [which is vegetarian]) or other stuffing mix

Prepare the stuffing according to the directions on the package and let it cool. In the meantime, coarsely grind the Quorn patties in a food processor. In a large bowl, thoroughly combine the Quorn mixture, chicken flavored broth, poultry seasoning, garlic powder, parmesan cheese, and breadcrumbs. Add the eggs and mix well. In a microwave/ oven-safe dish, mound the stuffing into the shape of a dome. Press down (using hands) until the stuffing feels slightly compact. Distribute the chicken mixture evenly over the stuffing, reserving enough mixture to shape into two drumsticks. Shape and place the drumsticks, arranged to look like wings. Microwave uncovered for 6 minutes on high power. Place in a preheated 350 degree oven for 15 minutes or until golden brown. If necessary, cover the bottom of the chicken to avoid premature browning.

Recipe by Marinés Fornerino

Truth be told, I am not so much a vegetarian as I am a fake-meat-atarian. I'm not really provegetables; I'm just anti-meat. And vegetarian staples that are dressed up to look, taste, smell, and feel like something they are not raise a particularly difficult issue in the study of food. There are not many examples of food items that are necessarily meant to represent other food items. Mashed potatoes are not typically served in the shape of mushrooms. Carrots are seldom processed in order to pass for asparagus. The fact that we take soybeans and make them look like meat is a particular and 
unique thing in the food world. One could argue, in fact, that the only food equivalent is animal flesh itself. That is, apart from steaks, ribs, and the like, we often go to great lengths to make meat on our plates look nothing like the animal that it truly was. The relationship between meatloaf and a cow, for instance, is very hard to see. A chicken McNugget looks nothing like a chicken. A fish stick looks nothing like a fish. And a hot dog looks nothing like...well...whatever a hot dog is made out of. Interesting, then, that we intentionally make our meat not resemble the animal, but make our tofu resemble meat. Fake meat products have even more in common with real meat than we might imagine, as both participate in artistic representation more than most other foods, intentionally offered up to be something that they are not.

Speaking of Meatloaf, there are, of course, non-Rocky Horror science fiction inspired examples of turning specific food stuffs into something that no longer resembles that food. It is important, for instance, that Soylent Green not look like people. I take it, in fact, to be the lesson of the movie "Soylent Green" (1973, Dir. Richard Fleischer) that cannibalism metaphorically stands in for the complete loss of a society's soul, the final nail in the coffin of our collective future. And in the movie, humans have so devastated the natural world-destroying all of the plants, animals, and ecosystems across the face of the earth-that there are no Others with whom to found a community, no life beyond human life to help construct our own sense of Self. Without the alterity, we implode and destroy ourselves.

This dystopic destiny is at odds with the more vegetarian future painted by "Star Trek." Vulcans, we are told, are vegetarians in the "Star Trek" universe, but so-at least to some extent- are most humans. This is because of a piece of technology called the replicator. Replicators are able to shape atoms in a specific pattern in order to recreate matter of any kind. If one stores the pattern for a cup of coffee-or for a steak or chicken breast or rack of ribs-in the computer's memory banks, the replicator can then assemble the necessary atoms to create an exact duplicate of that food item. This would be the ultimate in fake meat because such technology could create an identical copy of the real thing, yet no animals would be involved at all. Most people would still be eating "meat," but none of it would be real. It has to be admitted that this does seem better than what we are doing today, but is there still not something wrong with an entire culture that fashions atoms into animal flesh in order to satiate its desire for meat?

We are getting close to this future right now. In 2007, scientists were able to grow a healthy colony of muscle tissues in a test tube. In vitro meat. Granted, most of what has been accomplished so far has been created using home-grown muscle cells from a mouse, but one imagines that thick slabs of mouse muscle are likely to taste a little like chicken. If actual chicken is more to our tastes, then we 
need only note the recent success of a group in Australia which was able to grow enough cloned poultry muscle cells in order to process them into a small patty of actual chicken steak.

In the spring of 2008, PETA was so overjoyed at the possibility of cloned meat that they announced a one million dollar prize to the first person to come up with an economically viable way to grow meat in a test tube for mass consumption. The thinking is that just like with the "Star Trek" replicator, there will have to be one animal that suffers at the very start-one model who will provide the blueprint for all future meat of that type, one martyr for the cause-but then the suffering will end.

True. It will end. And we will all still be a society of meat-eaters.

Thus far there are only questions. So let us imagine one last thought experiment. Let us assume that you, the reader, are vegetarian, and one fine summer evening you are invited over to a cookout at a friend's house. Everyone in attendance is a vegetarian, and happy to be eating veggie hot dogs. It's like a real American BBQ with baseball, apple pie, and Chevrolets all around. The charcoal grill makes the veggie dogs plump and warm, and you are enjoying the company and the taste. Then, toward the end of the evening, the host announces that he has, all along, secretly been playing a rather horrible game with everyone. It's something he calls "Hot Dog Roulette," in which, hidden amongst all of the veggie dogs there was one real hot dog served to someone at some point in the evening. For many vegetarians, this would be traumatic. And we need to think this through: to think about why we might have thoroughly enjoyed the taste of the hot dogs that were passed out, commended them for their closeness to the real thing, and then felt outraged when that simulation was so good that we could not tell the difference between having eaten the fake and having eaten the real meat. How would we feel in relation to our desire? How would we come to grips with our complicity? Many vegetarians have, perhaps, mistakenly eaten animal flesh of some sort before, but this is more easily confessed and forgiven for most because they feel less responsibility if the transgression was born of ignorance. How would this be different if the transgression were born both of ignorance and a real desire for the real thing? Although we live in a post-Baudrillardian world of simulacra where the real and the simulation have collapsed into one, there is still a major difference between the real hot dog and the veggie dog for most of us. The question is, how might we deal with the ethics of our actions and our desires in a situation such as this?

Let us at least say this. We need to address two essential concerns, and I propose that phenomenology can help us on both counts. First, we must be clear about the relationship between the fake and the real; and second, we must find a way of describing the ethics of intentions and actions that does not 
rely on Kant or Mill, but instead gets at what lies beneath and ties together intentions and actions.

It seems that the fake and the real cannot be defined in terms of each other but only in terms of something else outside of the system that they comprise. We cannot say that the dichotomous realfake system is comprised of merely A and not-A. If we list all of the qualities of A, it is not the case that the second item-the fake-is just a negation of those qualities. In fact, each thing in such a pair has separate and distinct qualities. And so it would seem that in order to tell which is the real and which is the fake, we would have to compare those qualities with some sort of standard or ideal that stands outside of the system. This thinking is as old as the Greeks, but we must try to avoid, for the moment, all forms of Platonism. Further, this is also not a dialectic relationship. The fake and the real do not comprise the first two stages of some greater moment which is yet to come but which will synthesize them into something better and more rational. The more general problem is that to use the language of "the real" and "the fake" is already to adopt a metaphysics of correspondence where one thing corresponds truly and another falsely. And this, in turn, is based on the belief that there is something outside of experience, and that truth is established when belief lines up with this external reality. It is, in short, everything that phenomenology rejects.

In Husserlian terms we might say that the notion of "the fake" is founded on the faulty principle that there is only one way for a thing to appear truly. It is to say, for instance, that of the following shapes, each representing the way in which a rectangular table might be seen from some specific angle, only one is a true rectangle: the rest are distorted by perspective into parallelograms and trapezoids-into fake rectangles.
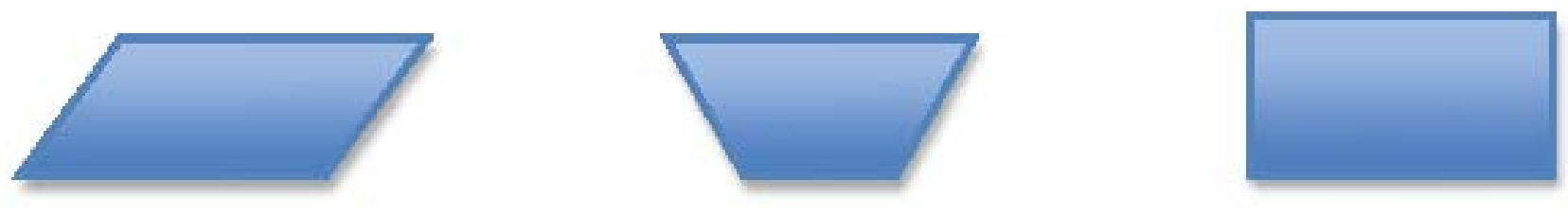

One of Husserl's most brilliant insights is that this is not true. These are all rectangles. They are just rectangles appearing from different vantage points. Each is precisely what we expect a rectangle to look like from some specific angle. This is what a rectangle is: all of the ways in which it can appear. A rectangle should look sort of squished and slanty when seen from an angle precisely in order to appear rectangular. Perspective is not a distortion; and subjectivity is not something to get around in our search for the truth.

To make matters clearer, Husserl invokes the metaphor of an horizon. The rectangular table appears 
within an horizon of explicit and implicit appearings. The horizon is infinite and stretches forever because there is always some new angle and some new context from which to see the table. No matter how we experience it now, there are other possible experiences we could still be having. The object can never be exhausted. And yet, it is something specific. Our experiences are grouped together in an identity synthesis that pulls the experiences together so that we realize they all have the same intentional object: the rectangular table. On the fringe of the horizon, the rectangular table will appear as a flat line. It might even appear as an oval under very specific and peculiar circumstances. But that is precisely what it means to be a rectangular table when seen under these conditions.

This is not to say that there is no such thing as an invalid judgment about the identity of an object. Sometimes we make mistakes. It's closing time in a store and one rushes toward a sales attendant with a purchase only to find out at the last moment that-in the bad lighting and from a distance-one made a mistake and took a mannequin for a real person. What we have learned in such a case is that, under the right conditions, a mannequin can appear as a person. And it is most likely that a person can appear as a mannequin. There is a sort of overlap in the horizons of the two things, though they are two distinct things. We have learned something about the Being of mannequins and the Being of persons.

And so: what of fake meat? Is a veggie dog something that is separate from a hot dog, or is it merely one way in which a hot dog can appear? We can no longer simply say that a veggie dog is a fake hot dog. But if we move beyond the discourse of "the fake and the real," can we still make distinctions in a meaningful way? Because sometimes it is definitely the fake thing that we crave and other times it is not the fake thing that we want.

Let us not say that the immorality of a situation is primarily in the desire, in the acting on the desire, or in the consequences of that action. Rather it is the phenomenological act of taking up an Other's bad as such as one's own good. Life is always already suffused with the ethical because Goods appear to us just as anything else appears to us. To be more precise, ethics is a sort of phenomenological categoriality. In the same way that buttered bread is more than just "bread plus butter"-in the same way that we can directly experience the being-together of the bread and the butter and the way in which this being-together "permeates" the whole experience, the way in which the buttered bread is presenced-so, too, do we experience the values that are inherent in objects, actions, and states of affairs. If you feed me when I am hungry, the experience is more than you + me + food. I can experience the ethical import of it all, the way in which you are taking up my good as such as your own, the way in which it is an expression of compassion or love. But what if you feed me a vegetarian hot dog? Is this a sort of love? 
We can, perhaps, tie everything together in this way. What is it we desire when we desire fake meat? The memory of having enjoyed meat in the past? What the taste represents? It is not clear that what we really desire is meat-dead animal flesh-itself. Intentions can be important, but ethics is more than this. If I intend to paint a picture of a lake, then the object of consciousness is the lake. The lake can be meant in many ways because there are many ways in which a thing can be present. Vegetarian meat is perhaps like art in this way: I intend to create a representation of something else-to make it present. I don't get wet in the painting of the lake although it is the lake that is present, but do I get my hands symbolically bloody with a veggie dog? Not necessarily. Because it is not necessarily a meat hot dog that is made present by a veggie dog. The horizons of separate things can overlap as we are pulled in two different directions. And the ethics with which the two things are suffused can be quite different as well.

We are not absolved of our wrong-doing by the complexity of appearance: it still all rests on our shoulders. And this is a conclusion that leaves things up in the air a bit; but it is, at least, to point in a direction toward which we can begin moving in hopes of addressing such an issue-as well as the larger questions of vegetarianism and animal-representation of all kinds. It is, I hope, thus a true conclusion rather than a false one. 